\title{
SINGULAR INTEGRALS ON NILPOTENT LIE GROUPS
}

\author{
ROBERT S. STRICHARTZ
}

ABSTRACT. Convolution operators $T f(x)=\int f\left(x y^{-1}\right) K(y) d y$ on a class of nilpotent $L_{i e}$ groups are shown to be bounded on $L^{p}, 1<p$ $<\infty$, provided the Euclidean Fourier transform of $K$ (with respect to a coordinate system in which the group multiplication is in a special form) satisfies familiar "multiplier" conditions.

1. Statement of results. The Calderón-Zygmund theory of singular integrals-convolutions on $\mathbf{R}^{n}$ with kernels homogeneous of degree - $n$-has been extended to the context of nilpotent Lie groups by Knapp and Stein [3] and Korányi and Vági [4]. The Euclidean theory, however, also deals with a larger class of operators-the so-called multiplier transformations-where the condition on the kernel is in terms of its Fourier transform. Our purpose is to extend these results to a class of nilpotent Lie groups. The results are somewhat schizoid because the convolutions involve the group structure while the conditions on the kernel involve the Euclidean Fourier transform. Thus the class of operators obtained will depend on the choice of coordinates on the group, and only special coordinate systems and groups will be considered.

Let $\pi$ be a nilpotent Lie algebra of dimension $n$ and $N=\exp \pi$ the associated connected simply-connected nilpotent Lie group. Following Knapp and Stein we suppose there exists a one-parameter group of automorphisms (called dilations) $\delta(t)$ such that $\pi$ has a coordinate system for which

$$
\delta(t) X=\left(t^{a} X_{1}, t^{a} X_{2}, \ldots\right), \quad X \in{ }^{\top},
$$

for positive reals $a_{1}, a_{2}, \ldots$. Without loss of generality we may assume $1=$ $a_{1} \leq a_{2} \leq \cdots$. We say that a system of coordinates on $N$ is compatible with $\delta_{t}$ if $\delta(t) x=\left(t^{a} x_{1}, t^{a} x_{2}, \ldots\right), x \in N$.

An example of a compatible coordinate system on $N$ may be obtained by giving the point $\exp x_{1} X_{1} \exp x_{2} X_{2} \ldots$ coordinates $\left(x_{1}, x_{2}, \ldots\right)$, where $\left(X_{1}, X_{2}, \ldots\right)$ is a basis for $\gamma_{\text {satisfying }}(1)$. We say that a system of co. ordinates for $N$ is affine if the group multiplication is given in the coordinates by

Received by the editors June 11,1974 and, in revi sed form, October 23, 1974. AMS (MOS) subject classifications (1970). Primary 43A80, 44A25, 22E30; Secondary 43A15, 22E 25 .

Key words and phrases. Singular integrals, nilpotent Lie group, $L^{p}$ boundedness, multiplier transformations. 


$$
(x y)_{j}=x_{j}+y_{j}+B_{j}(x, y)
$$

where each $B_{j}(x, y)$ is a bilinear function.

From now on we will only consider nilpotent Lie groups with dilations possessing affine coordinates compatible with the dilations, and we fix one such coordinate system. We give two examples:

(a) Let $N$ be the group of lower triangular $n \times n$ matrices (real or complex) with diagonal entries equal to one. Then $N$ has obvious affine coordinates $x_{i j}, 1 \leq j<i \leq n$, with multiplication law

$$
(x y)_{i j}=x_{i j}+y_{i j}+\sum_{i<k<j} x_{i k} y_{k j}
$$

compatible with dilations $(\delta(t) x)_{i j}=t^{i-j} x_{i j}$

(b) Suppose $\pi$ is 2 -stage nilpotent; i.e. $[\Re,[\Re, \Re]]=0$. Let $\pi_{2}=$ $[\Re, \Re]$ and let $\pi_{1}$ be any complementary subspace. Choose a basis $X_{1} \ldots$ $X_{p}$ for $\pi_{1}$ and $Y_{1}, \ldots, Y_{q}$ for $\pi_{2}$ and define coordinates on $N$ by assigning the point $\exp (x \cdot X+y \cdot Y)$ coordinates $(x, y)$. These are affine because the Campbell-Hausdorff formula reduces to $\exp A \exp B=\exp (A+B+1 / 2[A, B])$ and they are compatible with the dilations $\delta(t)(x, y)=\left(t x, t^{2} y\right)$.

Next we choose a norm function $\|x\|$ with the following properties:

(i) $\|\delta(t) x\|=t\|x\|$,

(ii) $\|x\|>0$ for $x \neq 0$,

(iii) $\|x\|$ is $C^{\infty}$ for $x \neq 0$,

(iv) $\left\|x^{-1}\right\|=\|x\|$.

The existence and basis properties of norm functions are discussed in Knapp and Stein [3]. Aside from the normalization (i) (here we follow (4) rather than (3)), any positive power of a norm is again a norm. We will use the following properties:

$$
\|x y\| \leq \gamma(\|x\|+\|y\|)
$$

for a fixed constant $\gamma$ (modified triangle inequality),

$$
|\{x:\|x\| \leq t\}|=c t^{n_{0}}
$$

(Lebesgue measure), where $n_{0}=a_{1}+a_{2}+\cdots$.

We consider the Fourier transform

$$
\hat{f}(\xi)=\int e^{i x \cdot \xi} f(x) d x
$$

and note

$$
(f(\delta(t) x))^{\wedge}(\xi)=t^{-n} 0 \hat{f}\left(\delta\left(t^{-1}\right) \xi\right) .
$$

The Fourier transform will depend on the choice of coordinates. We are interested in convolution operators 


$$
T f(x)=\int f\left(x y^{-1}\right) K(y) d y
$$

where $K$ is a tempered distribution, the integral being suitably interpreted.

Theorem. Suppose $\hat{K}(\xi)=m(\xi)$ is a bounded function satisfying

$$
\left|(\partial / \partial \xi)^{\beta} m(\xi)\right| \leq A\|\xi\|^{-a \cdot \beta}
$$

(here $\left.a \cdot \beta=a_{1} \beta_{1}+a_{2} \beta_{2}+\cdots\right)$ for sufficiently many derivatives. Then the aperator (6) is bounded on $L^{p}$ for $1<p<\infty$ and of weak type $(1,1)$ with norm depending only on $A$.

Remarks. Condition (7) is sufficient for the $L^{p}$ boundedness of the Euclidean convolution operator $f \rightarrow \int f(x-y) K(y) d y$ according to a theorem of Fabes and Rivière [1] generalizing the well-known theorem of Hörmander (see [2]). We will need a somewhat larger number of derivatives than for the Euclidean case in order to handle the case $p=2$.

2. Proof of Theorem. We choose a smooth partition of unity $1=$ $\sum_{-\infty}^{\infty} \phi_{j}(\xi)$ for $\xi \neq 0$ where $\phi_{j}(\xi)=\phi_{0}\left(\delta\left(2^{j}\right) \xi\right)$ and $\phi_{j}(\xi)$ is supported in $2^{-j-1} \leq\|\xi\| \leq 2^{-j+1}$. Let $m_{j}(\xi)=\phi_{j}(\xi) m(\xi)$, and define $K_{j}(x)$ by $\hat{K}_{j}(\xi)=$ $m_{j}(\xi)$. Note that $K_{j} \in L^{1}$ so that

$$
f * K_{j}(x)=\int f\left(x y^{-1}\right) K_{j}(y) d y
$$

is well defined for any $f \in L^{p}$. We shall prove the $L^{p}$ and weak-type estimates for the operators

$$
T_{N} f=\sum_{j=-N}^{N} f * K_{j}
$$

with norms independent of $N$. Since it is clear that $T_{N} f \rightarrow T f$ as $N \rightarrow \infty$ in the distribution sense for $f \in \mathcal{S}$, the estimates for $T f$ follow by routine arguments.

Now it is easy to show that $m_{j}(\xi)$ satisfies the same estimates as $m(\xi)$ (see Fabes and Rivière [1]). Because $m_{j}(\xi)$ has support in $2^{-j-1} \leq\|\xi\| \leq$ $2^{-j+1},(7)$ becomes

$$
\left|(\partial / \partial \xi)^{\beta} m_{j}(\xi)\right| \leq c A 2^{j(a \cdot \beta)}
$$

We first treat the case $p=2$. For this it suffices to show $\left\|f * K_{j} * \widetilde{K}_{k}\right\|_{2} \leq c A^{2} 2^{-|j-k|}\|f\|_{2}$ and $\left\|f * \widetilde{K}_{j} * K_{k}\right\|_{2} \leq c A^{2} 2^{-|j-k|}\|f\|_{2}$

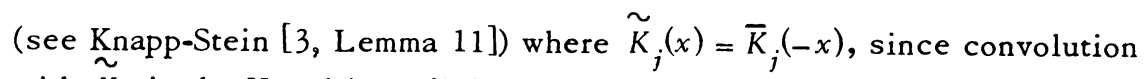
with $K_{j}$ is the Hermitian adjoint of convolution with $K_{j}$. We will actually establish 


$$
\left\|K_{j} * \widetilde{K}_{k}\right\|_{1} \leq c A^{2} 2-|j-k|
$$

and

$$
\left\|\tilde{K}_{j} * K_{k}\right\|_{1} \leq c A^{2} 2^{-|j-k|} .
$$

We now come to the heart of the proof-the actual computation of $K_{j} *$ $\tilde{K}_{k}$. We recall that the assumption that the coordinates are affine means

$$
(x y)_{j}=x_{j}+y_{j}+\sum_{k} B_{j k}(x) y_{k}
$$

where $B_{j k}(x)$ are linear functions. From the compatibility with dilations we obtain

$$
B_{j k}(\delta(t) x)=t^{{ }^{i} j-a}{ }^{-a} B_{j k}(x)
$$

in particular,

$$
B_{j k}(x)=0 \text { unless } a_{j} \geq a_{k}+1
$$

\section{Lemma 1.}

$$
K_{j} * \widetilde{K}_{k}(x)=\frac{1}{(2 \pi)^{n}} \int e^{-i x \cdot \xi} m_{j}(\xi) \overline{m_{k}(b(x, \xi))} d \xi
$$

where $b(x, \xi)_{r}=\xi_{r}+\Sigma_{s} B_{s r}(x) \xi_{s}$.

Proof. By the Fourier inversion formula,

$$
K_{j}(x y)=\frac{1}{(2 \pi)^{n}} \int e^{-i(x y) \cdot \xi} m_{j}(\xi) d \xi
$$

and

$$
(x y) \cdot \xi=\sum_{r} x_{r} \xi_{r}+\sum_{r} y_{r} \xi_{r}+\sum_{r} \sum_{s} P_{s r}(x) \xi_{s} y_{r}=x \cdot \xi+b(x, \xi) \cdot y .
$$

Thus

$$
\begin{aligned}
K_{j} * \ddot{K}_{k}(x) & =\int K_{j}(x y) \overline{K_{k}(y)} d y \\
& =\frac{1}{(2 \pi)^{n}} \iint e^{-i x \cdot \xi} m_{j}(\xi) \overline{e^{i b(x, \xi) \cdot y} K_{k}(y)} d \xi d y \\
& =\frac{1}{(2 \pi)^{n}} \int e^{-i x \cdot \xi} m_{j}(\xi) \overline{m_{k}(b(x, \bar{\xi}))} d \xi . \quad \text { Q.E.D. }
\end{aligned}
$$

Now we use the lemma to estimate $K_{j} * \widetilde{K}_{k}$. We observe first that the hypotheses on $m(\xi)$ are dilation invariant, so that by a simple homogeneity argument it suffices to estimate $K_{0} * \widetilde{K}_{j}$ and $K_{j} * \widetilde{K}_{0}$ for $j \leq 0$. Since these functions are obviously integrable, it suffices to obtain the estimate for $j$ $\leq-3$ where $m_{0}(\xi)$ and $m_{j}(\xi)$ have disjoint supports. Then 


$$
m_{0}(\xi) \overline{\left.m_{j}(b)(x, \xi)\right)}=m_{0}(\xi) \overline{\left(m_{j}(b(x, \xi))\right.}-\overline{\left.m_{j}(\bar{\xi})\right)}
$$

so

$$
\left|K_{0} * \widetilde{K}_{j}(x)\right| \leq \frac{1}{(2 \pi)^{n}} \int\left|m_{0}(\xi)\right|\left|m_{j}(b(x, \xi))-m_{j}(\xi)\right| d \xi
$$

The integral extends only over the support of $m_{0}(\xi)$, which is $1 / 2 \leq\|\xi\| \leq 2$, so it follows from (8) and the mean value theorem that

$$
\left|K_{0} * \widetilde{K}_{j}(x)\right| \leq c A^{2} 2^{j} \sup _{1 / 2 \leq\|\xi\| \leq 2}|b(x, \xi)-\xi| \leq c A^{2} 2^{j}\|x\|^{a_{n}-1}
$$

by (12) and (13). This gives the desired estimate for the integral over the region $\|x\| \leq 1$. Next we multiply (14) by a monomial $x^{\beta}$ and integrate by parts to obtain

$$
x^{\beta} K_{0} * K_{j}=\frac{(-i)|\beta|}{2 \pi}-\int e^{-i x \cdot \xi}\left(\frac{\partial}{\partial \xi}\right)^{\beta}\left(m_{0}(\xi) \overline{\left.m_{j}(\overline{b(x}, \bar{\xi})\right)} d \xi .\right.
$$

We expand the derivative in Leibniz' formula. The term $\overline{m_{j}(b(x, \xi))}$ $(\partial / \partial \xi)^{\beta} m_{0}(\xi)$ is estimated as before, while the remaining terms are treated more simply by observing that

$$
\left|(\partial / \partial \xi)^{\gamma} m_{j}(b(x, \xi))\right| \leq c A 2^{j}\|x\|^{\gamma \cdot a-|\gamma|}
$$

for $1 / 2 \leq|\xi| \leq 2$ by (12) and (13). Thus

$$
\left|x^{\beta} K_{0} * \widetilde{K}_{j}(x)\right| \leq c A^{2} 2^{j}\left(\|x\|^{a}{ }^{-1}+\|x\|^{\beta \cdot a-|\beta|}\right) .
$$

By taking $x^{\beta}=x_{i}^{\beta_{i}}$ for $\beta_{i}$ sufficiently large, and summing on $i$, we obtain (9). The estimate for $K_{j} * \tilde{K}_{0}$ is analogous since

$$
K_{j} * \widetilde{K}_{0}\left(x^{-1}\right)=\int K_{j}(y) \overline{K_{0}(x y)} d y,
$$

and the proof of (10) is essentially the same.

Next we prove the weak-type $(1,1)$ estimate. If we write $R_{N}=\Sigma_{-N}^{N} K_{j}$ it suffices to prove (see [4])

$$
\int_{\|y\| \geq 3 \gamma\|x\|}\left|R_{N}\left(x^{-1} y\right)-R_{N}(y)\right| d y \leq c A,
$$

where $y$ is the constant in (3) and $c$ is independent of $N$. Now the estimate

$$
\int_{\|y\| \geq 3 \gamma\|x\|}\left|R_{N}(y-x)-R_{N}(y)\right| d y \leq c A
$$

is known from the Euclidean case [1] so it suffices to show

$$
\int_{\|y\| \geq 3 \gamma\|x\|}\left|R_{N}(x-1 y)-R_{N}(y-x)\right| d y \leq c A .
$$


Actually the proof of (17) is a simple variant of the proof of (16) once we observe that, as a function of $y$, we can compute the Fourier transform of $K_{j}\left(x^{-1} y\right)-K_{j}(y-x)$ as

$$
\left(K_{j}\left(x^{-1} y\right)-K_{j}(y-x)\right)^{\wedge}(\xi)=e^{i x \cdot \xi}\left(m_{j}(b(x, \xi))-m_{j}(\xi)\right) .
$$

Now we choose integers $r_{1} \ldots r_{n}$ such that $r_{k} a_{k}>n_{0} / 2$ for $k=1$, $\ldots, n$. Then we find

$$
\int_{\|y\| \leq t}|f(y)| d y \leq c t^{\prime \prime} 0^{\prime 2}\|f\|_{2}
$$

and

$$
\int_{\|y\| \geq t}|f(y)| d y \leq c \sum_{k=1}^{n} t^{\left(n_{0} / 2\right)-r_{k}{ }^{a}{ }_{k}}\left\|y_{k}^{r}{ }_{k} f(y)\right\|_{2}
$$

by Schwartz' inequality. We use (20) and the Plancherel theorem to estimate

$$
\begin{gathered}
\int_{\|y\| \geq 3 \gamma\|x\|}\left|K_{j}\left(x^{-1} y\right)-K_{j}(y-x)\right| d y \leq 2 \int_{\|y\| \geq y^{\prime}\left\|_{x}\right\|}\left|K_{j}(y)\right| d y \\
\leq c \sum_{k=1}^{n}\|x\|^{\left({ }^{n} / 2\right)-r_{k}{ }^{a} k \|}\left\|\left(\frac{\partial}{\partial \xi_{k}}\right)^{r} m_{j}(\xi)\right\|_{2} \\
\leq c A \sum_{k=1}^{n}\left(2^{j} /\|x\|\right)^{r_{k}{ }^{a} k_{k}-\left(n_{0} / 2\right)},
\end{gathered}
$$

where the first inequality follows from (3) and the last from (8). We will use this estimate when $2^{j} /\|x\|$ is small.

Next we consider the range $\|x\| \leq \epsilon 2^{j}$ (the constant $\epsilon$ will be chosen later).

Iemma 2. If $f(y)=K_{j}\left(x^{-1} y\right)-K_{j}(y-x)$ then

$$
\|f\|_{2} \leq c 2^{-j n_{0} / 2}\left(\|x\| / 2^{j}\right)
$$

and

$$
\left\|y_{k}^{r} f(y)\right\|_{2} \leq c 2^{j\left(r_{k} a_{k}-\left(n_{0} / 2\right)\right)}\left(\|x\| / 2^{j}\right) .
$$

Proof. By the Plancherel formula and (18) we have

$$
\|f\|_{2}=c\left(\int\left|m_{j}(b(x, \xi))-m_{j}(\xi)\right|^{2} d \xi\right)^{1 / 2} .
$$

We choose $f$ small enough that

$$
\|b(x, \xi)-\xi\| \leq 2^{-j} / 4 \gamma .
$$

This implies (by (3)) that $m_{j}(b(x, \xi))$ vanishes unless $2^{-j-2} / \gamma \leq\|\xi\| \leq \gamma 2^{-j+2}$, 
so we may take this as the range of integration in (24). The integrand is estimated by the mean-value theorem (8), (12) and (13):

$$
\begin{aligned}
\left|m_{j}(b(x, \xi))-m_{j}(\xi)\right| & \leq \sum_{s} \sum_{k} B_{s k}(x) \xi_{s}\left\|\frac{\partial}{\partial \xi_{s}} m_{j}\right\|_{\infty} \\
& \leq c \sum_{s} \sum_{k}\left(\|x\| / 2^{j}\right)^{a}{ }^{-a} k \leq c\|x\| / 2^{j}
\end{aligned}
$$

since $\|x\| \leq \epsilon 2^{j}$. This establishes (22).

The proof of (23) is quite similar. We have

$$
\left\|y_{k}^{r}{ }_{k} f(y)\right\|_{2}=c\left(\int\left|\left(\frac{\partial}{\partial \xi_{k}}\right)^{r k}\left\{e^{i x \cdot \xi}\left(m_{j}(b(x, \xi))-m_{j}(\xi)\right)\right\}\right|^{2} d \xi\right)^{1 / 2},
$$

and the integrand is dominated by $c 2^{j r_{k} a_{k}}\|x\| / 2^{j}$. Q.E.D.

We may now use (19) and (20) with $t=2^{j}$ to conclude

$$
\int\left|K_{j}\left(x^{-1} y\right)-K_{j}(y-x)\right| d x \leq c A\|x\| / 2^{j}
$$

when $\|x\| \leq \epsilon 2^{j}$. Combining this with (21) yields

$$
\begin{aligned}
& \int_{\|y\| \geq 3 \gamma\|x\|}\left|R_{N}\left(x^{-1} y\right)-R_{N}(y-x)\right| d y \\
& \quad \leq c A\left(\sum_{\|x\| \geq \epsilon 2^{j}} \sum_{k=1}^{n}\left(\frac{2^{j}}{\|x\|}\right)^{r_{k}{ }^{a} k^{-\left(n_{0} / 2\right)}}+\sum_{\|x\| \leq \epsilon 2^{j}} \frac{\|x\|}{2^{j}}\right)
\end{aligned}
$$

which proves $(17)$ and the weak-type $(1,1)$ estimate.

The $L^{p}$ boundedness for $1<p \leq 2$ follows by interpolation. To obtain the $L^{p}$ boundedness for $\infty>p \geq 2$ we must show that the adjoint operator, which is convolution with $\widetilde{K}$, is bounded on $L^{p}$ for $1<p \leq 2$. Now $\widetilde{K}$ need not satisfy the hypotheses of the Theorem. However it is easy to verify that $f * \tilde{K}\left(x^{-1}\right)=\bar{K} * g(x)$ where $g(x)=f\left(x^{-1}\right)$. Since $\bar{K}$ does satisfy the hypotheses of the Theorem and there is no essential difference between convolu. tion on the left and right, we obtain the desired conclusion.

3. Remarks. It is not at all clear to what extent the class of operators satisfying the hypotheses of the Theorem depends on the choice of coordinates, or whether the class is closed under composition. The homogeneous singular integrals of Knapp-Stein which are sufficiently smooth will satisfy the hypotheses in any affine coordinate system.

Fairly standard techniques will allow one to extend the $L^{p}$ boundedness to operators of the form $T f(x)=\int f\left(x y^{-1}\right) K(x, y) d y$, where $K(x, y)$ satisfies the hypotheses of the Theorem for each fixed $x$ (along with certain conditions on $x$-derivatives depending on which techniques are used). 


\section{REFERENCES}

1. E. B. Fabes and N. M. Rivière, Singular integrals with mixed homogeneity, Studia Math. 27 (1966), 19-38. MR 35 \#683.

2. L. Hörmander, Estimates for translation invariant operators in $L^{p}$ spaces, Acta Math. 104 (1960), 93-140. MR $22 \# 12389$.

3. A. Knapp and E. M. Stein, Intertwining operators for semi-simple groups, Ann. of Math. (2) 93 (1971), 489-578.

4. A. Korányi and S. Vági, Singular integrals in homogeneous spaces and some problems of classical analysis, Ann. Scuol a Norm. Sup. Pisa 25 (1971), 575-648.

5. E. M. Stein, Singular integrals and differentiability properties of functions, Princeton Math. Ser., no. 30, Princeton Univ. Press, Princeton, N. J., 1970. MR 44 $\# 7280$. 14853

DEPARTMENT OF MATHEMATICS, CORNELL UNIVERSITY, ITHACA, NEW YORK 